\title{
МЕТОДИКА ОЦЕНКИ ВОЕННО-ЭКОНОМИЧЕСКОЙ ЭФФЕКТИВНОСТИ МЕРОПРИЯТИЙ ПО УПРАВЛЕНИЮ УСТОЙЧИВОСТЬЮ ВОЕННО- ЛОГИСТИЧЕСКОЙ СИСТЕМЫ
}

\section{A METHOD OF EVALUATION OF MILITARY AND ECONOMIC EFFECTIVENESS OF MEASURES OF SUSTAINABILITY MANAGEMENT OF A MILITARY LOGISTIC SYSTEM}

M. Plotnik

Summary. The paper contains a method of evaluation of military and economic effectiveness of measures of sustainability management of a military logistic system. Indices of military and economic efficiency are proposed. A method of their calculation is developed. A method of calculation of the integral index of military and economic efficiency is described. A scale of its values is proposed.

Keywords: sustainability, military logistic system, military economic efficiency.

\author{
Плотник Михаил Александрович \\ Помощник начальника учебного отделения, B/ч \\ 42984, 2. Саратов \\ Expest.on@mail.ru
}

Аннотация. В статье предложена методика оценки военно-экономической эффективности мероприятий по управлению устойчивостью военно-логистической системы. Предложены показатели военной и экономической эффективности и разработана методика их расчета. Описана методика расчета интегрального показателя военно-экономической эффективности. Предложена шкала его значений.

Ключевые слова: устойчивость, военно-логистическая система, военно-экономическая эффективность.

мической эффективности, рассчитанным по разным методикам. Фактически данная ситуация указывает на то, что сама сущность показателя военно-экономической эффективности пока не была однозначно определена. По этой причине необходимо выбрать ту методику, которая будет оптимально соответствовать задачам данного исследования.

Для расчета показателя военно-экономического эффекта используются два базовых подхода [5]:

1. Определение значения военно-экономического эффекта ВЭЭ путем свертки рассчитанных отдельно показателей военного эффекта ВЭ (под которым, как правило, понимается прирост качества функционирования военной организации) и экономического эффекта ЭЭ (чаще всего определяемого как снижение затрат военной организации, полученное благодаря тем мерам, военно-экономическая эффективность которых оценивается):

$$
\text { ВЭЭ }=\operatorname{comb}(\text { ВЭ,ЭЭ), }
$$

где $\operatorname{comb}(x)$ - оператор свертки [6]. 
Таблица 1. Подходы к оценке прироста устойчивости цепей поставок

\begin{tabular}{|l|l|}
\hline Абсолютный прирост устойчивости $\Delta \mathrm{Y}$ & Относительный прирост устойчивости $\mathrm{S}$ \\
\hline$\Delta \mathrm{y}=\mathrm{y}_{1}-\mathrm{y}_{0}$ & $S=\frac{\mathrm{y}_{1}}{\mathrm{y}_{0}}$ \\
\hline
\end{tabular}

Таблица 2. Подходы к оценке экономического эффекта мероприятий по управлению устойчивостью ЦП

\begin{tabular}{|l|l|}
\hline Абсолютное изменение затрат $\Delta 3$ & Относительное изменение затрат $И$ \\
\hline$\Delta 3=3_{1}-3_{0}$ & И $=\frac{3_{1}}{3_{0}}$ \\
\hline
\end{tabular}

Этот подход соответствует процедуре многокритериального оценивания. Важным достоинством такого подхода является то, что он позволяет получить интегральную оценку качества функционирования военно-логистической системы (ВЛС). Однако недостаток состоит в том, что полученный показатель, как правило, лишен прозрачного военного, технического и экономического смысла. Кроме того, он очень зависит от процедуры свертки частных показателей. Самой важной проблемой является то, что, как правило, для свертки частных показателей в формуле (1) используются веса (позволяющие учесть важность отдельного частного показателя), корректно определить которые в ряде случаев затруднительно;

2. Использование относительного показателя военно-экономической эффективности Э как отношения военного эффекта ВЭ к затратам 3 на его достижение:

$$
\ni=\frac{\mathrm{B} \ni}{3} .
$$

Удобство этого показателя состоит в том, что он соответствует особенностям функционирования военно-логистических систем: они направлены на обслуживание потребностей военной организации государства и его оборонно-промышленного комплекса (ОПК), и поэтому, с точки зрения потребителей услуг ВЛС, задача ВЛС состоит не в максимизации прибыли (что характерно для ВЛС, организованных гражданскими логистическими операторами), а в минимизации издержек на их функционирование. Кроме того, он обладает прозрачным военным и экономическим смыслом (он показывает, какие затрат необходимы для прироста военного эффекта на единицу). Наконец, он не требует сложной процедуры свертки частных показателей. С учетом описанных достоинств этого показателя в рамках данного исследования для принятия решений по управлению устойчивостью ЦП мы будем использовать именно военно-экономическую эффективность.
Важно уточнить, что для принятия решений о целесообразности проведения мероприятий по управлению устойчивостью ВЛС необходимо оценивать не военно-экономическую эффективность ВЛС, а военно-экономическую эффективность этих мероприятий, тогда как формула (2) направлена на оценку военно-экономической эффективности ВЛС. Таким образом, нам необходимо адаптировать формулу (2) к специфике наших задач.

Логичным выбором для оценки военного эффекта от проведения мероприятий по управлению устойчивости цепи поставок является прирост устойчивости ЦП. Для оценки этого прироста можно использовать абсолютные и относительные показатели (см. табл. 1, величина $\mathrm{У}_{0}$ соответствует устойчивости ЦП до проведения мероприятий по повышению устойчивости ЦП, а величина $\mathrm{У}_{l}-$ устойчивости ЦП после проведения мероприятий).

Более удобным мы считаем использование показателя относительного прироста устойчивости $S$, поскольку он позволяет сравнивать ВЛС разного масштаба. Отметим, что, хотя мы говорим о приросте устойчивости, на практике, в случае неэффективных мер по управлению устойчивостью ЦП, она может снизиться, и тогда прирост устойчивости будет отрицательным.

Для оценки затрат при расчете показателей эффективности обычно используется абсолютная величина затрат. Однако в нашем случае такой подход неприемлем, поскольку нам необходимо определить, как изменились затраты на обеспечение функционирования ВЛС в результате проведения мероприятий по повышению ее устойчивости. Таким образом, для оценки военно-экономической эффективности мероприятий по повышению устойчивости ВЛС мы будем использовать изменение затрат. Отметим, что речь идет именно об изменении затрат, а не об их снижении (поскольку 
проведение соответствующих мероприятий может потребовать как первоначальных инвестиций, например, в расширение логистических мощностей, так и роста операционных издержек, в частности, на оплату труда дополнительного персонала). Как и в случае с военным эффектом, изменение затрат может быть оценено как в абсолютном, так и в относительном выражении (см. табл. 2, 3 0 соответствует затратам на функционирование ВЛС до проведения мероприятий по повышениюее устойчивости, а $3_{1}$ - затратам после проведения этих мероприятий). Изменение затрат представляет собой показатель экономического эффекта.

Для сохранения единообразия методики мы считаем целесообразным использовать показатель относительного изменения затрат И (поскольку для оценки военного эффекта был выбран показатель относительного прироста устойчивости).

Обратим внимание специфику расчета показателя затрат (экономического эффекта). Как видно из табл. 2, абсолютный экономический эффект принимает положительное значение в том случае, если затраты возрастают (аналогично, относительный эффект также больше единицы в том случае, если величина 31 больше величины 30). Это противоречит традиционному подходу оценки экономического эффекта на основе величины затрат - он считается положительным в том случае, если затраты после проведения мероприятий снижаются (т.е. если возникает экономия). Это противоречие связано с тем, что в качестве обобщающего показателя военно-экономического эффекта мы используем военно-экономическую эффективность, для расчета которой необходима величина затрат, а не экономии.

Под затратами на функционирование ВЛС мы понимаем не совокупную величину затрат в течение всего срока ее эксплуатации, а затраты в течение определенного периода (месяца или года).

При оценке затрат необходимо учитывать их разновременной характер (как было сказано выше, проведение мероприятий по повышению устойчивости ЦП может потребовать первоначальных инвестиций). Для этого затраты $3_{1}$ необходимо определять по формуле приведенных затрат [5]:

$$
3_{1}=30+I \times E,
$$

\section{где}

30 - операционные затраты на обеспечение функционирования ЦП (в течение определенного периода) после проведения мероприятий по повышению устойчивости ЦП;
I - инвестиции, направленные на повышение устойчивости ЦП;

$E-$ норматив экономической эффективности (для военно-логистических систем его значение можно определить как величину, обратную сроку эксплуатации ВЛС).

В практике коммерческих предприятий для анализа разновременных затрат чаще всего применяется показатель чистого дисконтированного дохода [5]. Однако для поставленной нами задачи оценки военно-экономической эффективности мероприятий по повышению устойчивости ЦП он не подходит. Показатель чистого дисконтированного дохода является инструментом инвестиционного анализа и позволяет оценить окупаемость инвестиций в проект. При проведении мероприятий по повышению устойчивости ЦП задача обеспечить окупаемость этих мероприятий не ставится, в силу чего предпочтительным является показатель приведенных затрат.

Эффективность Э мероприятий по повышению устойчивости ЦП будет рассчитываться по формуле

$$
\ni=\frac{S}{\mathrm{U}}=\frac{\mathrm{y}_{1}}{\mathrm{y}_{0}} / \frac{3_{1}}{3_{0}} .
$$

В качестве приемлемой может рассматриваться та стратегия повышения устойчивости ЦП, для которой $\ni>1$. Это равнозначно выполнению условия

$$
\frac{\mathrm{y}_{1}}{\mathrm{y}_{0}}>\frac{3_{1}}{3_{0}} \text {. }
$$

Формула (4) показывает, что к реализации может быть рекомендована та стратегия, которая связана с повышением издержек функционирования ВЛС, при условии, что рост издержек компенсируется более высоким ростом устойчивости.

Из формул (3) и (4) очевидно, что военно-экономическая эффективность Э мероприятий по повышению устойчивости ЦП представляет собой отношение военно-экономической эффективности ЦП до проведения мероприятий $\ni_{0}$ и после них $\ni_{1}$ :

$$
\ni=\frac{S}{И}=\frac{\mathrm{y}_{1}}{\mathrm{y}_{0}} / \frac{3_{1}}{3_{0}}=\frac{\mathrm{y}_{1}}{3_{1}} / \frac{\mathrm{y}_{0}}{3_{0}}=\frac{\ni_{1}}{\exists_{0}} .
$$

На практике целесообразно ввести дополнительные ограничения на критерии принятия решения о выборе стратегии повышения устойчивости ЦП:

1. $S \geq S_{\min }$, где $S_{\min }$ - установленное органами военного управления минимально допустимое значение прироста устойчивости ВЛС. Если это условие не выполняется в анализируемой стратегии, то прирост устойчивости слишком мал для того, 
Таблица 3. Шкала оценки эффективности мероприятий по повышению устойчивости ЦП

\begin{tabular}{|c|c|c|c|}
\hline $\begin{array}{l}\text { Оценка проведения } \\
\text { мероприятий }\end{array}$ & $\begin{array}{l}\text { Основной } \\
\text { показатель }\end{array}$ & $\begin{array}{l}\text { Дополнительные } \\
\text { показатели }\end{array}$ & Комментарии \\
\hline Эффективное проведение & $\ni_{\phi} \geq \ni_{\mathrm{n}}$ & $S_{\phi} \geq S_{\pi}, И_{\phi} \geq И_{\pi}$ & $\begin{array}{l}\text { Фактические значения всех показателей не хуже } \\
\text { прогнозных. Поставленные цели достигнуты, } \\
\text { ВЛС функционирует в требуемом режиме. }\end{array}$ \\
\hline \multirow[b]{2}{*}{ Приемлемое проведение } & \multirow[b]{2}{*}{$\exists_{\phi} \geq \exists_{\mathrm{n}}$} & $S_{\phi} \geq S_{\pi}, h_{\phi}<\mathrm{U}_{\text {п }}$ & $\begin{array}{l}\text { Хотя военно-экономическая эффективность } \\
\text { и военный эффект проведения мероприятия } \\
\text { не хуже прогнозных, достигнуты они были } \\
\text { за счет чрезмерно высоких затрат. Необходима } \\
\text { проверка обоснованности затрат }\end{array}$ \\
\hline & & $S_{\phi}<S_{\text {п, }}$ И $_{\phi} \geq \mathrm{U}_{\mathrm{n}}$ & $\begin{array}{l}\text { Военно-экономическая эффективность не хуже } \\
\text { прогнозной, однако требуемый уровень } \\
\text { устойчивости ВЛС не достигнут. Необходима } \\
\text { проверка правильности планирования } \\
\text { и проведения мероприятий и достаточности } \\
\text { выделенного для них финансирования }\end{array}$ \\
\hline \multirow[t]{2}{*}{ Неэффективное проведение } & \multirow[t]{2}{*}{$\ni_{\phi}<\ni_{\text {п }}$} & $S_{\phi} \geq S_{\text {п, }} \mathrm{U}_{\phi}<\mathrm{U}_{\mathrm{n}}$ & $\begin{array}{l}\text { Хотя целевого значения военного эффекта } \\
\text { удалось достичь, прогнозные затраты } \\
\text { на проведение мероприятий были значительно } \\
\text { превышены, что не позволило достичь } \\
\text { целевого значения военно-экономической } \\
\text { эффективности. Необходима корректировка } \\
\text { мероприятий }\end{array}$ \\
\hline & & $S_{\phi}<S_{\text {п }}, h_{\phi} \geq \mathrm{h}_{\mathrm{n}}$ & $\begin{array}{l}\text { Хотя при проведении мероприятий удалось } \\
\text { обеспечить целевое значение экономического } \\
\text { эффекта, военный эффект ниже ожидаемого, } \\
\text { в результате чего военно-экономическая } \\
\text { эффективность ниже прогнозной. Необходима } \\
\text { корректировка мероприятий }\end{array}$ \\
\hline Провальное проведение & $\ni_{\phi}<\ni_{\text {п }}$ & $S_{\phi}<S_{\text {п, }} \mathrm{U}_{\phi}>\mathrm{U}_{\text {п }}$ & $\begin{array}{l}\text { Фактические значения всех показателей ниже } \\
\text { прогнозных. Прогнозный уровень затрат } \\
\text { превышен, при этом требуемого уровня } \\
\text { устойчивости и военно-экономической } \\
\text { эффективности достичь не удалось. Необходим } \\
\text { пересмотр стратегии обеспечения устойчивости } \\
\text { Влс }\end{array}$ \\
\hline
\end{tabular}

чтобы данную стратегию было целесообразно реализовывать;

2. $И \leq И_{\max }$, где $И_{\max }$ - установленное органами военного управления максимально допустимое значение прироста затрат на обеспечение роста устойчивости ВЛС. Если это условие не выполняется, то, даже если анализируемая стратегия позволяет существенно повысить устойчивость ВЛС, реализовывать ее нецелесообразно, поскольку она требует чрезмерно высоких затрат;

3. Э $\geq Э_{\min }$, где $Э_{\min }-$ установленное органами военного управления минимальное значение военно-экономической эффективности.

Эти условия должны выполняться в комплексе. Отбор оптимального варианта стратегии повышения устойчивости ВЛС должен осуществляться только из тех вариантов, которые соответствуют приведенным выше условиям.
Оптимальный вариант стратегии должен соответствовать следующему условию:

$$
\left\{\begin{array}{c}
\ni \rightarrow \max ; \\
S \geq S_{\min } ; \\
И \leq И_{\max } \\
\ni \geq Э_{\min } .
\end{array}\right.
$$

После проведения мероприятий по повышению устойчивости ВЛС необходимо оценить фактическую эффективность этих мероприятий. Эту оценку следует проводить как по показателю военно-экономической эффективности (который является основным), так и по показателям военного и экономического эффекта (которые позволяют уточнить информацию о состоянии ВЛС). Введем следующие обозначения:

1. $\exists_{n}, S_{n}, И_{n}$ - прогнозные значения показателей военно-экономической эффективности, военного и экономического эффекта; 
Таблица 4. Шкала значений показателя результативности проведения мероприятий по повышению устойчивости ЦП

\begin{tabular}{|l|l|}
\hline Значение показателя $\mathbf{R}$ & $\begin{array}{l}\text { Оценка проведения мероприятий по повышению } \\
\text { устойчивости ЦП }\end{array}$ \\
\hline$R=3$ & Эффективное проведение \\
\hline$R=2$ & Приемлемое проведение \\
\hline$R=1$ & Неэффективное проведение \\
\hline$R=0$ & Провальное проведение \\
\hline
\end{tabular}

2. $\ni_{\phi}, S_{\phi}, и_{\phi}-$ фактические значения показателей военно-экономической эффективности, военного и экономического эффекта.

Шкала для оценки фактической эффективности мероприятий по повышению устойчивости ЦП представлена в табл. 3.

Приемлемому уровню проведения мероприятий по повышению устойчивости ВЛС соответствуют две ситуации (см. табл. 4):

1. Требуемый уровень устойчивости достигнут (и, возможно, превышен), но затраты на получение этого результата выше прогнозных;

2. Затраты на проведение мероприятий по повышению устойчивости ВЛС не выше прогнозных, однако требуемый уровень устойчивости не достигнут.

По нашему мнению, первый вариант является более предпочтительным, чем второй. В случае первого варианта необходимо принять меры по оптимизации затрат (что в будущих периодах позволит достичь заданных экономических параметров). Напротив, при втором варианте необходимо определить, с чем связана проблема:

1. Организационные причины - неправильное планирование мероприятий или их неэффективное проведение из-за некомпетентности сотрудников;

2. Финансовые причины - недостаточный объем финансирования.

Эти причины могут сочетаться. После выявления причин необходимо принять меры по их устранению и скорректировать проведенные мероприятия.

Мы можем ввести три логические функции для оценки результативности мероприятий по повышению устойчивости ЦП по трем ключевым параметрам:

1. По военно-экономической эффективности LEM:
$L E M= \begin{cases}1, & \frac{\ni_{\Phi}}{\ni_{\Pi}} \geq 1 \\ 0, & \frac{\ni_{\Phi}}{\ni_{\text {п }}}<1\end{cases}$

2. По военному эффекту $L M$ :

$L M= \begin{cases}1, & \frac{S_{\Phi}}{S_{\mathrm{n}}} \geq 1 ; \\ 0, & \frac{\mathrm{y}_{\Phi}}{\mathrm{y}_{\text {п }}}<1 ;\end{cases}$

3. По экономическому эффекту $L E$ :

$$
L E= \begin{cases}1, & \frac{И_{\text {п }}}{И_{\phi}} \leq 1 \\ 0, & \frac{И_{\text {п }}}{И_{\phi}}>1 .\end{cases}
$$

Далее мы предлагаем построить общий показатель результативности $R$, который будет рассчитываться по формуле:

$$
R=L E M+L M+L E
$$

Как очевидно из изложенного выше, показатель $R$ принимает только целочисленные значения в диапазоне от 0 до 3. На основе значения показателя $\mathrm{R}$ можно оценить качество реализации стратегии повышения устойчивости ЦП (см. табл. 4).

\section{Наши выво $\Delta ы$}

- для военно-экономического обоснования мероприятий по повышению устойчивости ЦП следует использовать показатель военно-экономической эффективности;

- при оценке целесообразности проведения мероприятий по повышению устойчивости ЦП необходимо вводить ограничения на значения 
военного эффекта, экономического эффекта и военно-экономического эффекта;

- после проведения мероприятий по повышению устойчивости ЦП необходимо оценить их фактическую эффективность путем сравнения фактических значений показателей эффективности с прогнозными. Для обобщенного описания качества проведения мероприятий нами предложен целочисленный показатель результативности, позволяющий отнести качество проведения этих мероприятий к одному из четырех диапазоHOB

\section{ЛИТЕРАТУРА}

1. Абдурахманова Э.Э., Бабенков А.В., Курбанов А.Х. Методические подходы к оценке военно-экономического эффекта от цифровизации логистики в системе материально-технического обеспечения военных потребителей // Вопросы оборонной техники. Серия 16: Технические средства противодействия терроризму. - 2019. — № 5-6.- - . 54-62.

2. Богатырева С.В., Титов А.Б., Куприянова М.Ю. Экономическая эффективность как основа формирования управленческих решений // Экономика и менеджмент систем управления. - 2016. - № 2-1.-С. 116-122.

3. Болдырев 0.А., Козин М.Н. Методические подходы к оценке военно-экономического эффекта в результате внедрения технических инноваций в системе материального обеспечения войск (сил) // Наука Красноярья. - 2018. - Т. 7. — № 2.- С. 40-56.

4. Бычков А.В., Стулов С.В., Курбанов А.Х. Оценка военно-экономической эффективности применения логистического потенциала Коллективных сил оперативного реагирования // Ученые записки Крымского инженерно-педагогического университета. — 2017. — № 4. - C. 28 -33.

5. Жеишев Р.С., Никитин Ю.А. Оценка военно-экономической эффективности системы продовольственного обеспечения войск (сил) в Арктической зоне Российской Федерации // Экономический вектор. - 2020.— № 2.- С. 96-102.

6. Котляров И.Д. Проблемы оценки эффективности аутсорсинга // Вестник Института экономики Российской академии наук. - 2017. — № 6. - С. 8799.

7. Красовитов Р.А. Методика оценки военно-экономического эффекта, получаемого при внедрении технологий «Индустрии 4.0» в сфере вещевого обеспечения военных потребителей // Наука Красноярья.— 2018. - Т. 7. — № 2.—C. 57-76.

8. Курбанов А.Х. Экономико-математическая модель оценки организационно-экономической эффективности внедрения аутсорсинга // Проблемы экономики и управления нефтегазовым комплексом. - 2012.— № 2.- С. 40-44.

9. Курбанов А.Х., Клюкин Е.В. Обоснование целесообразности и оценка военно-экономического эффекта от применения бенчмаркинга в управлении вещевым имуществом военной организации // Современная наука: актуальные проблемы теории и практики. Серия: Экономика и право.— 2015. № 11-12.- C. 46-50.

10. Курбанов А. Х., Колобов Е.О. Обоснование инструментов и подходов оценки военно-экономической эффективности применения контрейлерных перевозок в интересах материального обеспечения войск (сил) // Экономика и предпринимательств0. — 2016. — № 9. — C. 74-78.

11. Курбанов А.Х., Кузнецов А.В. Методика оценки военно-экономической эффективности управления аэродромно-техническим обеспечением ведомственной авиации // Экономика и менеджмент систем управления. - 2016. — № 4. - C. 25-31.

12. Стулов С.В. Метод оценки военно-экономической эффективности комбинированной логистической системы военных потребителей за пределами Российской Федерации в условиях ликвидации чрезвычайных ситуаций // Технологии гражданской безопасности. - 2019. - Т. 16. - № 4. - С. 64-68.

13. Чулков Д.Н. Оценка экономической эффективности применения шаблонов бизнес-процессов // Экономика и предпринимательство.- 2015.№ 9-2.—C. 1014-1017.

(c) Плотник Михаил Александрович ( Expest.on@mail.ru ).

Журнал «Современная наука: актуальные проблемы теории и практики» 